\title{
System-adapted correlation energy density functionals from effective pair interactions
}

\author{
Paola Gori-Giorgi and Andreas Savin \\ Laboratoire de Chimie Théorique, CNRS, Université Pierre et Marie Curie, 4 Place Jussieu, F-75252 Paris, France
}

(Dated: November 8, 2018)

\begin{abstract}
We present and discuss some ideas concerning an "average-pair-density functional theory", in which the ground-state energy of a many-electron system is rewritten as a functional of the spherically and system-averaged pair density $f\left(r_{12}\right)$. These ideas are further clarified with simple physical examples. We then show that the proposed formalism can be combined with density functional theory to build system-adapted correlation energy functionals. A simple approximation for the unknown effective electron-electron interaction that enters in this combined approach is described, and results for the He series and for the uniform electron gas are briefly reviewed.
\end{abstract}

\section{INTRODUCTION}

Density Functional Theory (DFT) is nowadays the most widely used method for the calculation of electronic structure in both solid-state physics and quantum chemistry [1, 2, 3]. The accuracy of the results coming from a DFT calculation is limited by the approximate nature of the exchange and correlation energy $E_{x c}[n]$ and the associated potential, its functional derivative. While, at present, many algorithms for a very accurate or even exact exchange potential $v_{x}(\mathbf{r})$ and energy $E_{x}[n]$ are available [3, 4], better approximations for calculating accurate correlation energies $E_{c}[n]$ are still needed.

In a recent paper [5], we proposed an alternative approach to build the DFT correlation energy $E_{c}[n]$. The method consists in solving simple radial equations to generate the spherically and system-averaged pair density $f\left(r_{12}\right)$ along the so-called adiabatic connection. Besides its practical use for DFT, we realized that this method has many aspects that deserve to be better investigated, including the possibility for an alternative theory completely based on $f\left(r_{12}\right)$. In this work, we sketch the basic ideas for this alternative theory, we further discuss them with simple physical examples, and we deal with some of the aspects that went overlooked in Ref. 5]. The scope of this paper is to lay the rigorous foundations for an approach that will be further developed towards the construction of a self-consistent scheme combining the radial equations for $f\left(r_{12}\right)$ with the Kohn-Sham equations.

The paper is organized as follows. After defining the notation, we develop in Sec. IIII the formalism corresponding to a theory based on the spherically and system averaged pair density, reviewing at the same time the corresponding concepts for DFT. We hope that this parallel treatment will make it easier for the reader to familiarize with the new concepts. We then give, in Sec. IV some simple physical examples. Section $\nabla$ is devoted to explain how the ideas of Sec. III can be used to build correlation energy functionals for DFT. A simple, physically motivated, approximation for the unknown effective eletron-electron interaction that appears in our formalism is discussed in Sec. VI] where applications to twoelectron atoms and to the uniform electron gas are also briefly reviewed. The last section is devoted to conclu- sions and perspectives.

\section{DEFINITIONS}

We start from the standard $N$-electron hamiltonian (in Hartree atomic units, $\hbar=m=a_{0}=e=1$, used throughout)

$$
\begin{aligned}
H & =T+V_{e e}+V_{n e}, \\
T & =-\frac{1}{2} \sum_{i=1}^{N} \nabla_{i}^{2}, \\
V_{e e} & =\frac{1}{2} \sum_{i \neq j}^{N} \frac{1}{\left|\mathbf{r}_{i}-\mathbf{r}_{j}\right|}, \\
V_{n e} & =\sum_{i=1}^{N} v_{n e}\left(\mathbf{r}_{i}\right),
\end{aligned}
$$

where $v_{n e}$ is the external potential due to nuclei. Given $\Psi$, the ground-state wavefunction of $H$, we consider two reduced quantities that fully determine, respectively, the expectation values $\left\langle\Psi\left|V_{n e}\right| \Psi\right\rangle$ and $\left\langle\Psi\left|V_{e e}\right| \Psi\right\rangle$, i.e., the usual one-electron density

$$
n(\mathbf{r})=N \sum_{\sigma_{1} \ldots \sigma_{N}} \int\left|\Psi\left(\mathbf{r}, \mathbf{r}_{2}, \ldots, \mathbf{r}_{N}\right)\right|^{2} d \mathbf{r}_{2} \ldots d \mathbf{r}_{N}
$$

and the spherically and system-averaged pair density (APD), which is obtained as an integral of $|\Psi|^{2}$ over all variables but $r_{12}=\left|\mathbf{r}_{1}-\mathbf{r}_{2}\right|$,

$$
\begin{array}{r}
f\left(r_{12}\right)=\frac{N(N-1)}{2} \sum_{\sigma_{1} \ldots \sigma_{N}} \times \\
\int\left|\Psi\left(\mathbf{r}_{12}, \mathbf{R}, \mathbf{r}_{3}, \ldots, \mathbf{r}_{N}\right)\right|^{2} \frac{d \Omega_{\mathbf{r}_{12}}}{4 \pi} d \mathbf{R} d \mathbf{r}_{3} \ldots d \mathbf{r}_{N}
\end{array}
$$

where $\mathbf{r}_{12}=\mathbf{r}_{1}-\mathbf{r}_{2}$, and $\mathbf{R}=\frac{1}{2}\left(\mathbf{r}_{1}+\mathbf{r}_{2}\right)$. The function $f\left(r_{12}\right)$ is also known in chemistry as intracule density [6, 6, 8, 9, 10, 11, 12], and, when multiplied by the volume element $4 \pi r_{12}^{2} d r_{12}$, is proportional to the probability distribution for the electron-electron distance. We 
then have

$$
\begin{array}{r}
\left\langle\Psi\left|V_{n e}\right| \Psi\right\rangle=\int n(\mathbf{r}) v_{n e}(\mathbf{r}) d \mathbf{r} \\
\left\langle\Psi\left|V_{e e}\right| \Psi\right\rangle=\int \frac{f\left(r_{12}\right)}{r_{12}} d \mathbf{r}_{12}=\int_{0}^{\infty} \frac{f\left(r_{12}\right)}{r_{12}} 4 \pi r_{12}^{2} d r_{12}
\end{array}
$$

In the following text we will also deal with modified systems in which the external potential and/or the electronelectron interaction is changed. Thus, the notation $V_{e e}$ and $V_{n e}$ will be used to characterize the physical system, while the modified systems will be defined by $W$ and $V$, with

$$
\begin{aligned}
W & =\frac{1}{2} \sum_{i \neq j}^{N} w\left(\left|\mathbf{r}_{i}-\mathbf{r}_{j}\right|\right), \\
V & =\sum_{i=1}^{N} v\left(\mathbf{r}_{i}\right)
\end{aligned}
$$

where the pairwise interaction $w$ always depends only on $\left|\mathbf{r}_{i}-\mathbf{r}_{j}\right|$.

\section{FORMALISM}

In this section we present a "APD-functional theory" (APDFT) based on the function $f\left(r_{12}\right)$ highlighting, step by step, the analogies in reasoning with the derivation of standard DFT.

\section{A. DFT - The universal functional}

In standard DFT one defines a universal functional of the one-electron density $n$ as resulting from a constrained search over all the antisymmetric wavefunctions $\Psi$ that yield $n$ [13]

$$
\tilde{F}\left[n ; V_{e e}, T\right]=\min _{\Psi \rightarrow n}\left\langle\Psi\left|T+V_{e e}\right| \Psi\right\rangle
$$

or, more completely, as a Legendre transform [14]

$$
\begin{aligned}
F\left[n ; V_{e e}, T\right]= & \sup _{v}\left\{\min _{\Psi}\left\langle\Psi\left|T+V_{e e}+V\right| \Psi\right\rangle\right. \\
& \left.-\int n(\mathbf{r}) v(\mathbf{r}) d \mathbf{r}\right\} .
\end{aligned}
$$

In both Eqs. (11) and (12), the dependence on the electron-electron interaction (and on the kinetic energy operator $T$ ) has been explictly shown in the functional. The universality of the functional $F$ stems exactly from the fact that the e-e interaction is always $1 / r$ (and that $T$ is always the same).

The ground-state energy $E_{0}$ of the system can then be obtained by minimizing the energy with respect to $n$,

$$
E_{0}=\min _{n}\left\{F\left[n ; V_{e e}, T\right]+\int n(\mathbf{r}) v_{n e}(\mathbf{r}) d \mathbf{r}\right\}
$$

\section{B. APDFT - The system-dependent functional}

Similarly, we can define a system-dependent functional (i.e., a functional depending on the external potential $V_{n e}$, and thus on the specific system) of the APD $f\left(r_{12}\right)$ as

$$
\tilde{G}\left[f ; V_{n e}, T\right]=\min _{\Psi \rightarrow f}\left\langle\Psi\left|T+V_{n e}\right| \Psi\right\rangle,
$$

or better as

$$
\begin{aligned}
G\left[f ; V_{n e}, T\right]= & \sup _{w}\left\{\min _{\Psi}\left\langle\Psi\left|T+W+V_{n e}\right| \Psi\right\rangle\right. \\
& \left.-\int f\left(r_{12}\right) w\left(r_{12}\right) d \mathbf{r}_{12}\right\} .
\end{aligned}
$$

The ground-state energy can be obtained by a minimization with respect to $f$

$$
E_{0}=\min _{f}\left\{G\left[f ; V_{n e}, T\right]+\int \frac{f\left(r_{12}\right)}{r_{12}} d \mathbf{r}_{12}\right\} .
$$

Evidently, with respect to DFT, the functional $G$ has the disadvantage of being not universal: in DFT, an approximation for $F$ should be in principle valid for all systems. However, the crucial point for applications is understanding how difficult is to build a reasonable approximation for $G\left[f ; V_{n e}, T\right]$, given a certain $V_{n e}$. In the particular combination of DFT and APDFT that we propose in Sec. $\nabla$ the lack of universality of $G$ is not an issue.

Another issue concerns the $N$-representability conditions on $f\left(r_{12}\right)$, i.e., which contraints must a given $f$ satisfy to guarantee that it comes from the contraction of a $N$-electron wavefunction $\Psi$. This is a problem shared with other generalizations of DFT, like the pair-density functional theory [15, 16, 17, 18, 19]. The $N$-representability conditions on $f\left(r_{12}\right)$ are evidently related to those on the pair density, and we thus might expect that they are not a trivial matter [19]. The definition of the functional $G$ of Eq. (15) formally overcomes this problem by giving a divergent $(+\infty)$ answer for any non $N$-representable $f$ (when $f$ is not $N$-representable the right-hand side of Eq. (15) is not bounded from above), but this is not a practical solution when coming to applications. As we shall see, in the particular use of APDFT presented in Sec. $\nabla$ this issue is not particularly crucial from a practical point of view, since we use APDFT to build correlation functionals for DFT

\section{DFT - Adiabatic connection}

In density functional theory, one usually defines a set of hamiltonians depending on a parameter $\lambda$ [20, 21, 22],

$$
H^{\lambda}=T+W^{\lambda}+V^{\lambda},
$$

having all the same one-electron density, equal to the one of the physical system

$$
n^{\lambda}(\mathbf{r})=n(\mathbf{r}) \quad \forall \lambda
$$


If $W^{\lambda=0}=0$ and $W^{\lambda_{\text {phys }}}=V_{e e}$, one switches continuously from a noninteracting system to the physical system, while keeping the density fixed by means of a suitable external potential $V^{\lambda}$. Obviously, the APD $f\left(r_{12}\right)$ changes with $\lambda$. By the Hellmann-Feynmann theorem,

$$
\begin{array}{r}
\frac{\partial E_{0}^{\lambda}}{\partial \lambda}=\left\langle\Psi^{\lambda}\left|\frac{\partial W^{\lambda}}{\partial \lambda}+\frac{\partial V^{\lambda}}{\partial \lambda}\right| \Psi^{\lambda}\right\rangle= \\
=\int f^{\lambda}\left(r_{12}\right) \frac{\partial w^{\lambda}\left(r_{12}\right)}{\partial \lambda} d \mathbf{r}_{12}+\int n(\mathbf{r}) \frac{\partial v^{\lambda}(\mathbf{r})}{\partial \lambda} d \mathbf{r},
\end{array}
$$

so that by directly integrating Eq. (19), and by combining it with Eq. [13), one obtains

$$
F\left[n ; V_{e e}, T\right]=T_{s}[n]+\int_{0}^{\lambda_{\text {phys }}} d \lambda \int d \mathbf{r}_{12} f^{\lambda}\left(r_{12}\right) \frac{\partial w^{\lambda}\left(r_{12}\right)}{\partial \lambda},
$$

where $T_{s}[n]$ is the kinetic energy of a noninteracting system of $N$ electrons with density $n(\mathbf{r})$.

More generally, one can be interested in using as a starting point a system of partially interacting electrons, corresponding to a particular value of the coupling $\lambda$ (say, $\lambda=\mu$ ) between 0 and $\lambda_{\text {phys }}$. In this case, if $\Psi^{\mu}$ is the wavefunction of the system with partial interaction $W^{\mu}$ (and external potential $V^{\mu}$ ) we have

$$
\begin{aligned}
F\left[n ; V_{e e}, T\right]= & \left\langle\Psi^{\mu}\left|T+W^{\mu}\right| \Psi^{\mu}\right\rangle+ \\
& \left.+\int_{\mu}^{\lambda_{\text {phys }}} d \lambda \int d \mathbf{r}_{12} f^{\lambda}\left(r_{12}\right) \frac{\partial w^{\lambda}\left(r_{12}\right)}{\partial \lambda} 21\right)
\end{aligned}
$$

Usually, the adiabatic connection is performed along a linear path by setting $W^{\lambda}=\lambda V_{e e}$ (thus $\lambda_{\text {phys }}=1$ ), but some nonlinear choices can be more convenient when dealing with approximations.

\section{APDFT - Adiabatic connection}

We can also define a set of hamiltonians

$$
H^{\xi}=T+W^{\xi}+V^{\xi}
$$

in which the function $f\left(r_{12}\right)$ is kept fixed, equal to the one of the physical system,

$$
f^{\xi}\left(r_{12}\right)=f\left(r_{12}\right) \quad \forall \xi
$$

If $V^{\xi=0}=0$ and $V^{\xi_{\text {phys }}}=V_{n e}$, we are switching continuously from a system of $N$ free electrons interacting with a modified potential $w^{\xi=0}\left(r_{12}\right)$, to the physical system. That is, $f\left(r_{12}\right)$ is kept fixed as $\xi$ varies by means of a suitable electron-electron interaction $W^{\xi}$ while the one-electron density $n(\mathbf{r})$ changes with $\xi$. Again, by the Hellmann-Feynmann theorem, we find

$$
\begin{array}{r}
\frac{\partial E_{0}^{\xi}}{\partial \xi}=\left\langle\Psi^{\xi}\left|\frac{\partial W^{\xi}}{\partial \xi}+\frac{\partial V^{\xi}}{\partial \xi}\right| \Psi^{\xi}\right\rangle= \\
=\int f\left(r_{12}\right) \frac{\partial w^{\xi}\left(r_{12}\right)}{\partial \xi} d \mathbf{r}_{12}+\int n^{\xi}(\mathbf{r}) \frac{\partial v^{\xi}(\mathbf{r})}{\partial \xi} d \mathbf{r}
\end{array}
$$

so that

$$
G\left[f ; V_{n e}, T\right]=T_{\mathrm{f}}[f]+\int_{0}^{\xi_{\text {phys }}} d \xi \int d \mathbf{r} n^{\xi}(\mathbf{r}) \frac{\partial v^{\xi}(\mathbf{r})}{\partial \xi},
$$

where $T_{\mathrm{f}}[f]$ is the kinetic energy of a system of $N$ free fermions (zero external potential) having the same $f\left(r_{12}\right)$ of the physical system. A simple example of such adiabatic connection is given in Sec. IVA As we shall see, given a confined physical system, the corresponding $w^{\xi=0}\left(r_{12}\right)$ must be partially attractive (in order to create a bound cluster of fermions). This could in principle lead to "exotic" ground states for some of the systems corresponding to $\xi<\xi_{\text {phys }}$. This issue is not considered in this paper, and will be investigated in future work.

Similarly to the DFT case, it could be convenient to choose as starting point a system with an external potential corresponding to some coupling constant $\xi$ (say, $\xi=\alpha$ ) between 0 and $\xi_{\text {phys }}$. If $\Psi^{\alpha}$ is the ground-state wavefunction of the system with external potential $V^{\alpha}$ (and e-e interaction $W^{\alpha}$ ) we have

$$
\begin{aligned}
G\left[f ; V_{n e}, T\right]= & \left\langle\Psi^{\alpha}\left|T+V^{\alpha}\right| \Psi^{\alpha}\right\rangle+ \\
& +\int_{\alpha}^{\xi_{\text {phys }}} d \xi \int d \mathbf{r} n^{\xi}(\mathbf{r}) \frac{\partial v^{\xi}(\mathbf{r})}{\partial \xi} .
\end{aligned}
$$

\section{E. DFT - Kohn-Sham equations}

One-particle equations in DFT can be obtained by defining a set of orthogonal orbitals $\varphi_{i}(\mathbf{r})$ with occupation number $\nu_{i}$ that minimize $\sum_{i} \nu_{i}\left\langle\varphi_{i}\left|-\frac{1}{2} \nabla^{2}\right| \varphi_{i}\right\rangle$ and yield the density of the physical system, $\sum_{i} \nu_{i}\left|\varphi_{i}(\mathbf{r})\right|^{2}=$ $n(\mathbf{r})$. This gives

$$
\begin{aligned}
{\left[-\frac{1}{2} \nabla^{2}+v_{1}(\mathbf{r})\right] \varphi_{i}(\mathbf{r}) } & =\epsilon_{i} \varphi_{i}(\mathbf{r}) \\
\sum_{i} \nu_{i}\left|\varphi_{i}(\mathbf{r})\right|^{2} & =n(\mathbf{r}),
\end{aligned}
$$

where the potential $v_{1}(\mathbf{r})$ is the Lagrange parameter for the density. To fully specify these equations one needs a rule for the occupation $\nu_{i}$ of the orbitals. The KohnSham choice corresponds to occupy the orbitals in the same way as for a Slater determinant. This determinant is the wavefunction of a system of $N$ non-interacting electrons constrained to have the same one-electron density of the physical system, and leads to the identification

$$
T_{s}[n]=\min _{\left\{\varphi_{i}\right\} \rightarrow n} \sum_{i}\left\langle\varphi_{i}\left|-\frac{1}{2} \nabla^{2}\right| \varphi_{i}\right\rangle,
$$

with the same $T_{s}[n]$ of Eq. (20). The ground-state energy of the physical system is then obtained via the Hartreeexchange-correlation functional $E_{\mathrm{Hxc}}[n]$, defined as the difference $F\left[n ; V_{e e}, T\right]-T_{s}[n]$. This also implies that, in Eqs. (27), $v_{1}(\mathbf{r})=v_{\mathrm{KS}}(\mathbf{r})=v_{n e}(\mathbf{r})+\delta E_{\mathrm{Hxc}}[n] / \delta n(\mathbf{r})$.

Usually, the Kohn-Sham equations are derived starting from the noninteracting system with density $n(\mathbf{r})$, rather 
than from a constrained minimization of $\sum_{i} \nu_{i}\left\langle\varphi_{i}\right|-$ $\frac{1}{2} \nabla^{2}\left|\varphi_{i}\right\rangle$. This different way of proceeding allows us to keep the analogy with what we will do in the next subsection for APDFT.

\section{F. APDFT - effective equations}

Since the e-e interaction is spherically symmetric, the relevant APD that determines $\left\langle\Psi\left|V_{e e}\right| \Psi\right\rangle$ is a unidimensional quantity. To obtain simple "two-particle" equations for $f\left(r_{12}\right)$ we start from the kinetic energy operator for the scalar relative coordinate $r_{12}=\left|\mathbf{r}_{2}-\mathbf{r}_{1}\right|$,

$$
T_{12}=-\nabla_{r_{12}}^{2}=-\frac{1}{r_{12}} \frac{d^{2}}{d r_{12}^{2}} r_{12}+\frac{\ell(\ell+1)}{r_{12}^{2}}
$$

and we perform a minimization of $\sum_{i} \vartheta_{i}\left\langle\psi_{i}\left|-\nabla_{r_{12}}^{2}\right| \psi_{i}\right\rangle$ with respect to some orthogonal "effective" geminals $\psi_{i}\left(r_{12}\right)$ constrained to yield $f$ of the physical system, $\sum_{i} \vartheta_{i}\left|\psi_{i}\right|^{2}=f$, leading to

$$
\begin{aligned}
& {\left[-\nabla_{r_{12}}^{2}+w_{\mathrm{eff}}\left(r_{12}\right)\right] \psi_{i}\left(r_{12}\right)=\epsilon_{i} \psi_{i}\left(r_{12}\right)} \\
& \sum_{i} \vartheta_{i}\left|\psi_{i}\left(r_{12}\right)\right|^{2}=f\left(r_{12}\right) .
\end{aligned}
$$

The interaction $w_{\text {eff }}\left(r_{12}\right)$ is the Lagrange parameter for $f$. Again, to fully specify these equations we need a rule for the occupancy $\vartheta_{i}$ of the effective geminals. For spin compensated systems, we can choose to apply a rule that resembles to a Slater determinant: occupancy 1 for even $\ell$ (singlet symmetry), occupancy 3 for odd $\ell$ (triplet symmetry), up to $N(N-1) / 2$ occupied geminals. This rule has been applied to solve the effective equations (30) in the uniform electron gas, with rather accurate results [23, 24, 25]. It is however important to point out that when we apply this occupancy rule to Eqs. (30)

1. there is no Slater determinant that can be associated with our effective geminals: the $\psi_{i}$ are constrained to give the exact $f$ that cannot be obtained from a noninteracting wavefunction (for example, any Slater determinant violates the cusp condition satisfied by the exact $f$ );

2. more generally, there is no wavefunction (and so no physical system) that can be built from our effective geminals.

This last point implies that, if we define

$$
T_{g}[f]=\min _{\left\{\psi_{i}\right\} \rightarrow f} \sum_{i}\left\langle\psi_{i}\left|-\nabla_{r_{12}}^{2}\right| \psi_{i}\right\rangle
$$

(with the determinant-like occupancy), we have in general

$$
T_{g}[f] \neq T_{\mathrm{f}}[f],
$$

where $T_{\mathrm{f}}[f]$ was defined in Eq. (25). The total energy of the physical system can then be recovered via the kinetic and external-potential functional defined in Ref. [5], $F_{\mathrm{KE}}\left[f ; V_{n e}\right]=G\left[f ; V_{n e}, T\right]-T_{g}[f]$. This also leads to the identification, in Eqs (30), $w_{\text {eff }}\left(r_{12}\right)=$ $1 / r_{12}+\delta F_{\mathrm{KE}}\left[f ; V_{n e}\right] / \delta f\left(r_{12}\right)$.

An important issue to be addressed concerning the effective equations (30) is whether a given physical (and thus $N$-representable) $f\left(r_{12}\right)$ is also representable by the simple "effective-geminal" decomposition of Eqs. (30). This question is similar to the one arising in DFT: is a physical density always non-interacting representable? In view of the more complex nature of $f\left(r_{12}\right)$ with respect to $n(\mathbf{r})$ we might expect that this problem is much more difficult to face in APDFT than in DFT. It seems reasonable that at least the short-range part of a physical $f\left(r_{12}\right)$ is representable by Eqs. (30), while the long-range tail of $f$ of an extended system could be problematic [26].

\section{SIMPLE PHYSICAL EXAMPLES: TWO-ELECTRON SYSTEMS}

In this section we give some examples for two-electron systems, in order to gain physical insight with some of the ideas just introduced.

\section{A. A picture from harmonic forces}

A very simple picture of the whole adiabatic connection path in both DFT and APDFT can be gained by looking at an analytic-solvable model, i.e., a two-electron hamiltonian with only harmonic forces (harmonic electronnucleus attraction, and harmonic e-e repulsion too) [1],

$H(K, k)=-\frac{1}{2} \nabla_{1}^{2}-\frac{1}{2} \nabla_{2}^{2}+\frac{1}{2} K r_{1}^{2}+\frac{1}{2} K r_{2}^{2}-\frac{1}{2} k\left|\mathbf{r}_{1}-\mathbf{r}_{2}\right|^{2}$,

where $K>0$ (attractive nucleus-electron potential), $k>0$ (repulsive e-e interaction), and $K>2 k$, to have a bound system. For this hamiltonian,

$$
\begin{aligned}
n(r) & =\frac{2 \beta^{3 / 2}}{\pi^{3 / 2}} e^{-\beta r^{2}}, & \beta & =\frac{2 \sqrt{K(K-2 k)}}{\sqrt{K-2 k}+\sqrt{K}} \\
f\left(r_{12}\right) & =\frac{\gamma^{3 / 2}}{\pi^{3 / 2}} e^{-\gamma r_{12}^{2}}, & \gamma & =\frac{1}{2} \sqrt{K-2 k} .
\end{aligned}
$$

\section{DFT}

If our "physical" system corresponds to some $K=K_{n e}$ and $k=k_{e e}$, and we want to switch off the e-e interaction (by setting, e.g., $k=\lambda k_{e e}$ ) while keeping the density fixed, we will simply have to change $K_{n e}$ into $K(\lambda)$ such that $\beta$ in Eq. (34) does not change. The function $K(\lambda)$ is shown in the upper panel of Fig. 1 for the case $K_{n e}=3$, $k_{e e}=1$. We see that, as $\lambda \rightarrow 0, K(\lambda)$ decreases, because a smaller attraction is needed to keep the electrons in the density when there is no e-e repulsion. Of course, 
$f\left(r_{12}\right)$ changes with $\lambda$, as shown in in the lower panel of Fig. [1] as $\lambda$ decreases, the "on-top" value $f^{\lambda}\left(r_{12}=0\right)$ gets larger. This reflects the fact that when there is no e-e repulsion, it is more likely to find the two electrons close to each other.

\section{2. $A P D F T$}

If, instead, we switch off the external potential, (e.g., by setting $K=\xi K_{n e}$ ) while keeping $f\left(r_{12}\right)$ fixed, we will have to change the e-e interaction in order to keep $\gamma$ of Eq. (35) constant,

$$
k(\xi)=\frac{1}{2}(\xi-1) K_{n e}+k_{e e} .
$$

The one-electron density along this adiabatic connection is

$$
\begin{aligned}
n^{\xi}(r) & =\frac{2 \beta(\xi)^{3 / 2}}{\pi^{3 / 2} e^{-\beta(\xi) r^{2}}}, \\
\beta(\xi) & =\frac{2 \sqrt{\xi K_{n e}\left(K_{n e}-2 k_{e e}\right)}}{\sqrt{K_{n e}-2 k_{e e}}+\sqrt{\xi K_{n e}}} .
\end{aligned}
$$

Thus $n^{\xi}(r)$ is a gaussian that, as $\xi \rightarrow 0$, becomes more and more spread, as shown in the lower panel of Fig. 2] When the external potential goes to zero, the system becomes translationally invariant and the wavefunction for the center-of-mass degree of freedom is simply a plane wave. Correspondingly, the electron-electron interaction changes with Eq. (36): we see from the upper panel of Fig. 2 that, as $\xi$ gets smaller, $k(\xi)$ becomes smaller (less repulsive), and then it changes sign at some $0<\xi<$ 1 , becoming an attractive interaction. For a confined system, when the external potential approaches zero, an attractive e-e interaction is needed in order to keep $f\left(r_{12}\right)$ fixed. Moreover, in the very special case of harmonic forces there is a value $\xi^{*}=1-2 k_{e e} / K_{n e} \in(0,1)$ for which the e-e effective interaction is zero everywhere, $w^{\xi^{*}}=0$.

Of course, when the e-e physical interaction is the Coulomb potential $1 / r_{12}$ this cannot happen: a system with the same $f\left(r_{12}\right)$ of the physical system cannot have $w=0$ everywhere. This can be simply understood by thinking that there is no external potential that can force the system to have the correct cusp [27] at $r_{12}=0$. In fact, along any adiabatic connection that keeps $f\left(r_{12}\right)$ fixed, equal to the one of a system with Coulombic e-e interaction, $w^{\xi}\left(r_{12}\right)$ will always behave as $1 / r_{12}$ in the limit of small $r_{12}$.

\section{B. He atom}

Consider now the two electrons of a He atom. They feel the attraction of the nucleus, $-2 / r$, and they repel each other with potential $1 / r_{12}$. Given the exact (or a very accurate [28]) ground-state wavefunction $\Psi$, we can calculate the "exact" density $n(\mathbf{r})$ and the "exact" $f\left(r_{12}\right)$.
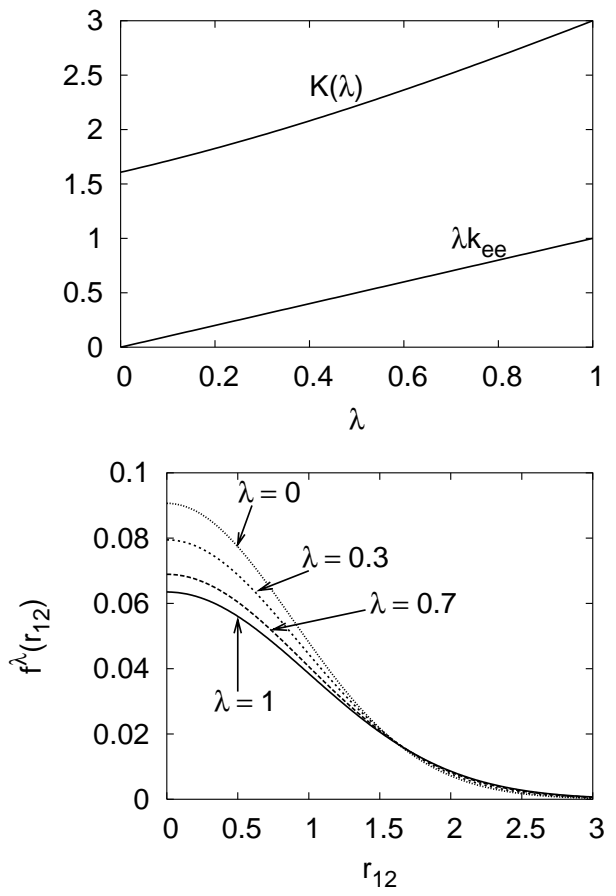

FIG. 1: Adiabatic connection in DFT for the simple harmonic hamiltonian of Eq. (33). The e-e interaction is multiplied by a parameter $\lambda$, and the density is kept fixed by a suitable external potential (upper panel). The APD $f\left(r_{12}\right)$ changes with $\lambda$ as shown in the lower panel.
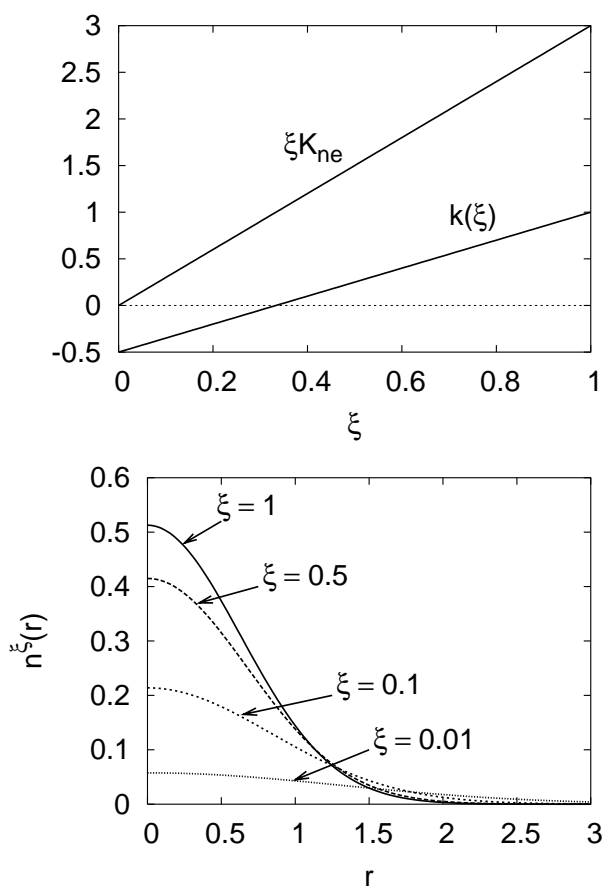

FIG. 2: Adiabatic connection in APDFT for the simple harmonic hamiltonian of Eq. (33). The external potential is multiplied by a parameter $\xi$, and the function $f\left(r_{12}\right)$ is kept fixed by a suitable electron-electron interaction (upper panel). The density $n(r)$ changes with $\xi$ as shown in the lower panel, and as $\xi \rightarrow 0$ becomes completely delocalized. 
Now, we can consider the case in which $W=0$ and $n(\mathbf{r})$ is kept fixed (the KS system in DFT, Sec. (IIC), and the one in which $V=0$ and $f\left(r_{12}\right)$ is kept fixed (APDFT, Sec. IIID) :

\section{DFT}

We construct a system which has the same density $n(\mathbf{r})$ of the physical one and no electron-electron interaction. This is the KS system, in which the two electrons do not interact $(w=0)$ and feel an external potential $v(r)$ less attractive than $-2 / r$, as in the case of the harmonic potential of Fig. 1 The APD $f^{\lambda=0}\left(r_{12}\right)$ of this system will be different from the physical one, as shown in Fig. 3 We see that the change in the function $f$ when we switch from the physical system to the KS one is qualititatively similar to the one of Fig. 11 i.e., at $\lambda=0$ the "on-top" value is higher than the physical one. In the case of Coulomb e-e interaction the physical $f\left(r_{12}\right)$ has a cusp, $f^{\prime}(0)=f(0)$, due to the short-range divergence of $1 / r_{12}$ [27].

\section{2. $A P D F T$}

In APDFT, we can construct a system which has the same $f\left(r_{12}\right)$ of the physical one, and zero external potential $(V=0)$. This is a system of two bounded fermions interacting with the effective potential $w^{\xi=0}\left(r_{12}\right)$ of Fig. 4 (calculated from an accurate [5, 28] $f$ ). As in the case of harmonic forces, the density of this system is completely delocalized, because the wavefunction for the center-ofmass degree of freedom is a plane wave. We can imagine that along the linear adiabatic connection, $v^{\xi}(r)=$ $-2 \xi / r$, the corresponding $w^{\xi}\left(r_{12}\right)$ changes smoothly between $1 / r_{12}$ (at $\left.\xi=\xi_{\text {phys }}=1\right)$ and the potential $w^{\xi=0}\left(r_{12}\right)$ of Fig. 目 As anticipated, we se that at $\xi=0$ the effective e-e interaction has an attractive part, which is necessary to have the same $f$ of a physical confined system. However, as already pointed out, for small $r_{12}$, the e-e interaction must always behave as $1 / r_{12}$, to produce the exact cusp in $f\left(r_{12}\right)$ [27].

\section{FROM APDFT TO CORRELATION ENERGY FUNCTIONALS FOR DFT}

In Sec.III we have underlined the similarity of the roles played by $n(\mathbf{r})$ and $f\left(r_{12}\right)$ from a mathematical point of view: the former completely determines $\left\langle\Psi\left|V_{n e}\right| \Psi\right\rangle$, and the latter $\left\langle\Psi\left|V_{e e}\right| \Psi\right\rangle$. However, while the KS system is a substantial simplification of the many-electron problem (yielding to single particle equations), the auxiliary system of Sec. IIID (with zero external potential) is still a complicated many-body object, consisting of $N$ fermions interacting with a partially attractive potential. The radial equations of Sec. ஹIF are a great simplification of

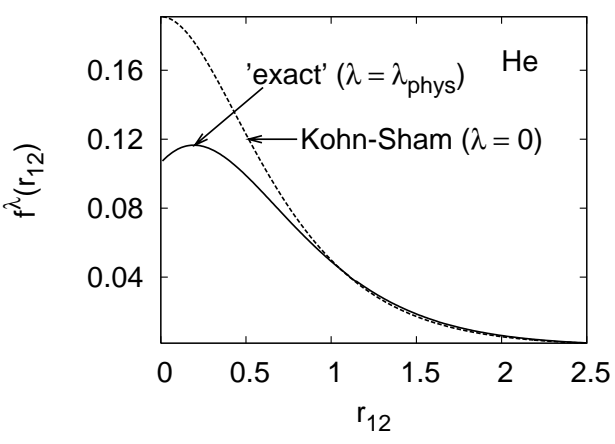

FIG. 3: The function $f^{\lambda}\left(r_{12}\right)$ at the two ends of the adiabatic connection in DFT for the He atom. For $\lambda=0$, we have a system of two noninteracting $(W=0)$ electrons constrained by an external potential to yield the same density of the physical system. For $\lambda=\lambda_{\text {phys }}$ we have the physical system with full interaction $1 / r_{12}$ and external potential $-2 / r$ (from the wavefunction of Ref. 28]; see also Ref. [5]).

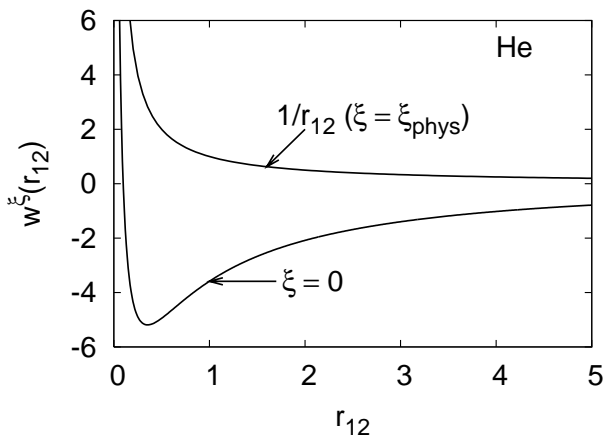

FIG. 4: The electron-electron interaction $w^{\xi}\left(r_{12}\right)$ at the two ends of the adiabatic connection in APDFT for the He atom. For $\xi=0$ we have a system of two free fermions (zero external potential) interacting with $w^{\xi=0}\left(r_{12}\right)$. This system has the same $f\left(r_{12}\right)$ of the physical system, but a completely delocalized one-electron density. For $\xi=\xi_{\text {phys }}$ we have the physical system, with e-e interaction $1 / r_{12}$ and external potential $-2 / r$. (The potential $w$ at $\xi=0$ has been calculated from the accurate wavefunction of Ref. [28]; see Ref. [5] for more details.)

the problem, but we might expect that building approximations for the whole functional $F_{\mathrm{KE}}\left[f ; V_{n e}\right]$ could be not easy.

Our basic idea, instead, is to use APDFT to build what is missing in DFT, i.e., to build $f^{\lambda}\left(r_{12}\right)$ along the adiabatic connection in DFT. As said in the Introduction, we insert our approach in the framework of exact-exchange DFT [3, 4, 29, 30] in which only the correlation energy functional needs to be approximated. The ground-state energy of the physical system is given by

$$
E_{0}=T_{s}[n]+\int n(\mathbf{r}) v_{n e}(\mathbf{r}) d \mathbf{r}+E_{\mathrm{H}}[n]+E_{x}[n]+E_{c}[n],
$$

where $E_{\mathrm{H}}[n]$ is the usual Hartree term, $E_{x}[n]$ is the ex- 
change energy, obtained by putting the Kohn-Sham orbitals in the Hartree-Fock expression for exchange, and the correlation energy, $E_{c}[n]$, is unkown. Equation (38) can be also rewritten as

$$
E_{0}=\left\langle\Phi_{\mathrm{KS}}\left|T+V_{e e}+V_{n e}\right| \Phi_{\mathrm{KS}}\right\rangle+E_{c}[n],
$$

where $\Phi_{\mathrm{KS}}$ is the Slater determinant of Kohn-Sham orbitals, i.e., the wavefunction of $N$ noninteracting electrons constrained to yield the same $n(\mathbf{r})$ of the physical system. Combining Eq. (20) with Eq. (39), we see that the wanted correlation energy is given by

$$
E_{c}[n]=\int_{0}^{\lambda_{\text {phys }}} d \lambda \int_{0}^{\infty} d r_{12} 4 \pi r_{12}^{2} f_{c}^{\lambda}\left(r_{12}\right) \frac{\partial w^{\lambda}\left(r_{12}\right)}{\partial \lambda},
$$

where

$$
f_{c}^{\lambda}\left(r_{12}\right)=f^{\lambda}\left(r_{12}\right)-f^{\lambda=0}\left(r_{12}\right)=f^{\lambda}\left(r_{12}\right)-f_{\mathrm{KS}}\left(r_{12}\right) .
$$

Thus, in order to get the KS correlation energy, we should compute $f^{\lambda}\left(r_{12}\right)$ for each hamiltonian $H^{\lambda}$ of Sec. IIIC Our approach consists in solving the simple radial equations of Sec. IIIF for each $H^{\lambda}$ along the adiabatic connection in DFT. This is not particularly expensive: we are dealing with unidimensional equations, and, if the dependence of $w^{\lambda}$ on $\lambda$ is smooth, we will only need few $\lambda$ values $(\sim 5-30)$ between 0 and $\lambda_{\text {phys }}$. With this particular combination of APDFT and DFT, we do not need to approximate the whole functional $F_{\mathrm{KE}}$ along the DFT adiabatic connection, but only its functional derivative, i.e., the effective interaction $w_{\text {eff }}\left(r_{12}\right)$ which appears in Eqs. (30), since the remaining information is provided by DFT. As we shall see, simple physical arguments can be used to build reasonable approximations for $w_{\text {eff }}\left(r_{12}\right)$ at each coupling strength $\lambda$.

Since the effective equations yielding $f_{c}^{\lambda}\left(r_{12}\right)$ must be solved for each system, we speak of system-adapted correlation energy density functionals.

\section{BUILDING APPROXIMATIONS}

In Ref. [5], we proposed and successfully tested a simple approximation for building $w_{\text {eff }}\left(r_{12}\right)$ along the DFT adiabatic connection for two-electron atoms. This approximation starts from $w_{\text {eff }}^{(0)}\left(r_{12}\right)$, the effective e-e interaction that gives $f_{\mathrm{KS}}\left(r_{12}\right)$ when inserted in Eqs. (30). In the special case of two-electron systems, $w_{\text {eff }}^{(0)}\left(r_{12}\right)$ is directly available in a KS calculation. For systems with more than two electrons, $w_{\text {eff }}^{(0)}\left(r_{12}\right)$ could be calculated, e.g., with the methods of Refs. 31, 32]. Then, the idea is to build an approximation for a correlation potential, to be added to $w_{\text {eff }}^{(0)}\left(r_{12}\right)$, which describes the change in $f$ when the e-e interaction is turned on, from zero to $w^{\lambda}\left(r_{12}\right)$. To do this, we defined an average density $\bar{n}$,

$$
\bar{n}=\frac{1}{N} \int d \mathbf{r} n(\mathbf{r})^{2},
$$

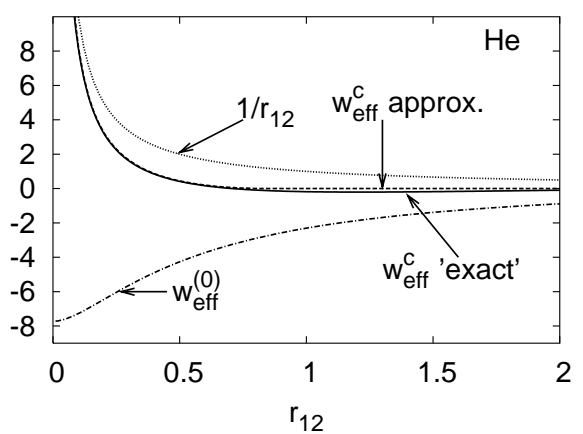

FIG. 5: Construction of an approximation for the effective potential that generates the APD $f\left(r_{12}\right)$ of the He atom: $w_{\text {eff }}^{(0)}$ is the part of the potential that generates the APD of the Kohn-Sham system. The "exact" [5, 28] correlation potential $w_{\text {eff }}^{c}$ and our approximation of Eq. (45) are shown, together with the Coulomb repulsion $1 / r_{12}$.

and, correspondingly, an average radius $\bar{r}_{s}$,

$$
\bar{r}_{s}=\left(\frac{4 \pi}{3} \bar{n}\right)^{-1 / 3} .
$$

We then built a correlation potential $w_{\text {eff }}^{c, \lambda}\left(r_{12}\right)$, as

$$
w_{\text {eff }}^{c, \lambda}\left(r_{12}\right)=w^{\lambda}\left(r_{12}\right)-\int_{|\mathbf{r}| \leq \bar{r}_{s}} \bar{n} w^{\lambda}\left(\left|\mathbf{r}-\mathbf{r}_{12}\right|\right) d \mathbf{r} .
$$

The idea behind Eq. (44) is the following: the e-e interaction $w^{\lambda}$ is screened by a sphere of radius $\bar{r}_{s}$ and of positive uniform charge of density $\bar{n}$ that attracts the electrons with the same interaction $w^{\lambda}$. The average density $\bar{n}$ of Eq. (42) (and thus the average $\bar{r}_{s}$ ) is kept fixed to mimic the fact that the one-electron density does not change along the adiabatic connection.

In order to gain insight with our construction, let us consider the case of the physical system, $w^{\lambda=\lambda_{\text {phys }}}=$ $1 / r_{12}$, for which Eq. (44) corresponds to

$$
w_{\mathrm{eff}}^{c}\left(r_{12}\right)=\left(\frac{1}{r_{12}}+\frac{r_{12}^{2}}{2 \bar{r}_{s}^{3}}-\frac{3}{2 \bar{r}_{s}}\right) \theta\left(\bar{r}_{s}-r_{12}\right),
$$

where $\theta(x)$ is the Heaviside step function. In Fig. [5 we reported, for the He atom, the potential $w_{\text {eff }}^{(0)}$ which generates $f_{\mathrm{KS}}$, together with the "exact" correlation potential $w_{\text {eff }}^{c}$, and the approximation of Eq. (45). We see that the potential $w_{\text {eff }}^{(0)}$ is a confining potential for the variable $r_{12}$ : our idea is to include in this term, available from DFT, the contribution to $f\left(r_{12}\right)$ coming from the particular external potential of the system and from the fermionic structure of the wavefunction. The remaining part to be approximated, the correlation potential $w_{\text {eff }}^{c}$, must include the effect of the e-e repulsion while keeping the density fixed, i.e., it must be essentially a screened Coulomb interaction. We see from Fig. 5 that the simple approximation of Eq. 45) is reasonable, i.e., the screening length is well approximated by $\bar{r}_{s}$ of Eqs. (42)-(43). 
For comparison, the full Coulomb repulsion $1 / r_{12}$ is also shown. Notice that in the special case of two-electron systems we have $T_{g}[f]=T_{\mathrm{f}}[f]$, so that the potential $w^{\xi=0}$ of Fig. 4 corresponds, in Fig. 5 , to the sum of $w_{\text {eff }}^{(0)}$ and the "exact" $w_{\text {eff }}^{c}$.

In Ref. [5], we inserted the potential $w_{\text {eff }}^{(0)}\left(r_{12}\right)+$ $w_{\text {eff }}^{c, \lambda}\left(r_{12}\right)$ into Eqs. (30), and solved them for several twoelectron atoms. Our results can be summarized as follows: (i) at $\lambda=\lambda_{\text {phys }}$ (i.e., for $\left.w^{\lambda}\left(r_{12}\right)=1 / r_{12}\right)$ we obtained APD $f\left(r_{12}\right)$ in close agreement with those coming from accurate variational wavefunctions [28], especially at small $r_{12}$; (ii) by setting $w^{\lambda}\left(r_{12}\right)=\operatorname{erf}\left(\lambda r_{12}\right) / r_{12}$ (the "erf" adiabatic connection), the KS correlation energies from Eq. (40) have an error which is less than $4 \mathrm{mH}$ for nuclear charges $Z \geq 2$; (iii) again with the "erf" adiabatic connection, we found that when the reference system corresponds to some $\lambda=\mu$ between zero and $\lambda_{\text {phys }}$ [as in Eq. (21)] our correlation energies are one order of magnitude better for $\mu \gtrsim 1 / \bar{r}_{s}$.

The correlation potential of Eq. (45), originally proposed by Overhauser [33], has been also used to solve the effective Eqs. (30) for the uniform electron gas (UEG), yielding to a very accurate description of the short-range part of $f\left(r_{12}\right)$ at all densities [23]. A more sophisticated effective potential, based on a self-consistent Hartree approximation, extended such accuracy to the long-range part of the UEG $f\left(r_{12}\right)$ at metallic densities 24]. Other simple approximations for $w_{\text {eff }}\left(r_{12}\right)$ in the UEG have also been proposed and tested [25].

\section{CONCLUSIONS AND PERSPECTIVES}

We have presented the ideas concerning a theory based on the spherically and system-averaged pair den- sity $f\left(r_{12}\right)$, and we have suggested to combine it with DFT to obtain system-adapted correlation energy functionals. So far, the method has been tested for the $\mathrm{He}$ series [5] and for the uniform electron gas [23, 24, 25], yielding promising results.

In order to completely develop the approach presented here, many steps are still to be performed and many issues are to be addressed. Among them, the most relevant ones concern the construction of better approximations for the effective electron-electron interaction that enters the formalism, and the implementation of a selfconsistent scheme to combine the Kohn-Sham equations with the correlation energy functional arising from our approach. Last but not least, with the approximations tested so far our approach works very well for the shortrange part of $f\left(r_{12}\right)$, so that the combination with multideterminantal DFT [21, 34] (in which only the shortrange correlations are treated within DFT) is also very promising and deserves further investigation.

\section{Acknowledgments}

We thank C. Umrigar for the wavefunctions of Ref. 28]. This research was supported by a Marie Curie Intra-European Fellowships within the 6th European Community Framework Programme (contract number MEIF-CT-2003-500026).
[1] W. Kohn, Rev. Mod. Phys. 71, 1253 (1999).

[2] A.E. Mattsson, Science 298, 759 (2002).

[3] C. Fiolhais, F. Nogueira, and M. Marques (eds.), A Primer in Density Functional Theory (Springer-Verlag, Berlin, 2003).

[4] see, e.g., S. Kümmel and J.P. Perdew, Phys. Rev. B 68, 035103 (2003); W. Yang and Q. Wu, Phys. Rev. Lett. 89, 143002 (2002); R. J. Magyar, A. Fleszar, and E. K. U. Gross, Phys. Rev. B 69, 045111 (2004); M. Grüning, O. V. Gritsenko, and E. J. Baerends, J. Chem. Phys. 118, 7183 (2003).

[5] P. Gori-Giorgi and A. Savin, Phys. Rev. A 71, 032513 (2005).

[6] A.J. Thakkar, A.N. Tripathi, and V.H. Smith, Jr., Int. J. Quantum Chem. 26, 157 (1984), and references therein.

[7] C.A. Coulson and A.H. Neilson, Proc. Phys. Soc. London 78, 831 (1961).

[8] J. Cioslowski, B.B. Stefanov, A. Tan, and C.J. Umrigar, J. Chem. Phys. 103, 6093 (1995).

[9] J. Cioslowski and G. Liu, J. Chem. Phys. 109, 8225
(1998).

[10] E. Valderrama, J.M. Ugalde, and R.J. Boyd, in Manyelectron densities and reduced density matrices, edited by J. Cioslowski (Kluwer Academic/Plenum Publishers, New York, 2000).

[11] E.R. Davidson, Reduced Density Matrices in Quantum Chemistry (Academic Press, New York, 1976).

[12] A.J. Coleman and V.I. Yukalov, Reduced Density $M a_{-}$ trices: Coulson's Challenge (Springer-Verlag, New York, 2000).

[13] M. Levy, Proc. Natl. Acad. Sci. U.S.A. 76, 6062 (1979).

[14] E. Lieb, Int. J. Quantum Chem. 24, 243 (1983).

[15] P. Ziesche, Phys. Lett. A 195, 213 (1994); M. Levy and P. Ziesche, J. Chem. Phys. 115, 9110 (2001).

[16] A. Gonis, T.G. Schulthess, J. van Ek, and P.E.A. Turchi, Phys. Rev. Lett. 77, 2981 (1996); A. Gonis, T.G. Schulthess, P.E.A. Turchi, and J. van Ek, Phys. Rev. B 56, 9335 (1997).

[17] A. Nagy, Phys. Rev. A 66, 022505 (2002).

[18] F. Furche, Phys. Rev. A 70, 022514 (2004). 
[19] P.W. Ayers, submitted to J. Math. Phys.

[20] W. Yang, J. Chem. Phys. 109, 10107 (1998).

[21] A. Savin, F. Colonna, and R. Pollet, Int. J. Quantum Chem. 93, 166 (2003), and references therein.

[22] J. Harris and R. Jones, J. Phys. F 4, 1170 (1974); D.C. Langreth and J.P. Perdew, Solid State Commun. 17, 1425 (1975); O. Gunnarsson and B.I. Lundqvist, Phys. Rev. B 13, 4274 (1976).

[23] P. Gori-Giorgi and J.P. Perdew, Phys. Rev. B 64, 155102 (2001).

[24] B. Davoudi, M. Polini, R. Asgari, and M.P. Tosi, Phys, Rev. B 66, 075110 (2002).

[25] M. Corona, P. Gori-Giorgi, and J.P. Perdew, Phys. Rev. B 69, 045108 (2004); I. Nagy, R. Diez Muiño, J.I. Juaristi, and P.M. Echenique, Phys. Rev. B 69, 233105 (2004).

[26] P. Ziesche, to appear in Phys. Status Solidi B.

[27] A. K. Rajagopal, J. C. Kimball, and M. Banerjee, Phys. Rev. B 18, 2339 (1978); X.-Y. Pan and V. Sahni, J. Chem. Phys. 119, 7083 (2003).

[28] D.E. Freund, B.D. Huxtable, and J.D. Morgan III, Phys. Rev. A 29, 980 (1984). We used an improved version (provided to us by C. Umrigar) of the accurate varia- tional wavefunctions described in this work to obtain oneelectron densities $n(\mathbf{r})$ and functions $f\left(r_{12}\right)$. See also C.J. Umrigar and X. Gonze, Phys. Rev. A 50, 3827 (1994), and Ref. [8].

[29] M. Seidl, J.P. Perdew, and S. Kurth, Phys. Rev. Lett. 84, 5070 (2000).

[30] J.P. Perdew, A. Ruzsinszky, J. Tao, V.N. Staroverov, G.E. Scuseria, and G.I. Csonka, J. Chem. Phys., to appear.

[31] F. Colonna and A. Savin, J. Chem. Phys. 110, 2828 (1999).

[32] Q. Zhao, R. C. Morrison, and R. G. Parr, Phys. Rev. A 50, 2138 (1994); R. van Leeuwen and E. J. Baerends Phys. Rev. A 49, 2421 (1994).

[33] A.W. Overhauser, Can. J. Phys. 73, 683 (1995).

[34] A. Savin, in Recent Developments and Applications of Modern Density Functional Theory, edited by J.M. Seminario (Elsevier, Amsterdam, 1996); T. Leininger, H. Stoll, H.-J. Werner, and A. Savin, Chem. Phys. Lett. 275, 151 (1997); R. Pollet, A. Savin, T. Leininger, and H. Stoll, J. Chem. Phys. 116, 1250 (2002); J. Toulouse, F. Colonna, and A. Savin, Phys. Rev. A 70, 062505 (2004). 